\title{
Papel del péptido mitocondrial humanina como blanco terapéutico en cáncer y neurodegeneración
}

\author{
Role of mitochondrial-derived peptide humanin as a therapeutic target in \\ cancer and neurodegeneration
}

Antonela Sofía Asad ${ }^{1}$, Camila Florencia Zuccato ${ }^{1}$, Alejandro Javier Nicola Candia ${ }^{1}$, María Florencia Gottardo ${ }^{2}$, Mariela Alejandra Moreno Ayala ${ }^{3}$, María Susana Theas ${ }^{4}$, Adriana Seilicovich ${ }^{5}$, Marianela Candolfi ${ }^{6}$

\section{Resumen}

La humanina es un péptido derivado de la mitocondria con efectos protectores robustos contra una gran variedad de estímulos citotóxicos en diversos tipos celulares. Esto la convierte en un blanco terapéutico interesante para muchas enfermedades, como el cáncer y enfermedades neurodegenerativas, entre otras. Además, este péptido podría utilizarse como un biomarcador en estas enfermedades. Durante la última década, han sido desarrollados análogos y péptido-miméticos de la humanina que muestran resultados prometedores en modelos preclínicos. A su vez, también se está explorando el potencial terapéutico de vectores de terapia génica que puedan sobreexpresar o silenciar la humanina endógena. Varios puntos importantes a considerar antes de trasladar estas estrategias terapéuticas a la clínica son su posible papel en la progresión del cáncer y la eventual generación de quimiorresistencia. Todos estos temas serán abordados en este artículo de revisión.

Palabras claves: Humanina; péptido mitocondrial; cáncer; enfermedades neurodegenerativas.

\footnotetext{
1 Estudiantes de doctorado del Consejo Nacional de Investigaciones Científicas y Técnicas en la Universidad de Buenos Aires. Especialistas en inmunoterapia y terapia génica contra el glioblastoma y cáncer de mama en el Instituto de Investigaciones Biomédicas (INBIOMED, UBA-CONICET), Facultad de Medicina, Universidad de Buenos Aires, Buenos Aires, Argentina.

2 Estudiante de postdoctorado del Consejo Nacional de Investigaciones Científicas y Técnicas en la Universidad de Buenos Aires.

Especialista en oncología en el Laboratorio de Oncología Molecular de la Universidad de Quilmes, Bernal, Argentina.

3 Estudiante de postdoctorado. Especialista en oncología del Departamento de Biología Celular y Molecular, University of California,

Berkeley, CA, USA.

4 Investigadora independiente del Consejo Nacional de Investigaciones Científicas y Técnicas. Especialista en fertilidad masculina en el Instituto de Investigaciones Biomédicas (INBIOMED, UBA-CONICET), Facultad de Medicina, Universidad de Buenos Aires, Buenos Aires, Argentina.

5 Investigadora Superior del Consejo Nacional de Investigaciones Científicas y Técnicas. Especialista en endocrinología en el Instituto de Investigaciones Biomédicas (INBIOMED, UBA-CONICET), Facultad de Medicina, Universidad de Buenos Aires, Buenos Aires, Argentina 6 Investigadora Independiente del Consejo Nacional de Investigaciones Científicas y Técnicas. Especialista en inmunoterapia y terapia génica contra el glioblastoma y cáncer de mama en el Instituto de Investigaciones Biomédicas (INBIOMED, UBA-CONICET), Facultad de Medicina, Universidad de Buenos Aires, Buenos Aires, Argentina.
}

ORCID:

https://orcid.org/0000-0002-1234-7250 - Antonela Sofía Asad

https://orcid.org/0000-0002-3170-7397 - Camila Florencia Zuccato

https://orcid.org/0000-0001-6599-5094 - María Florencia Gottardo

https://orcid.org/0000-0002-7612-6852 - Mariela Alejandra Moreno Ayala

https://orcid.org/0000-0002-8971-9833 - María Susana Theas

https://orcid.org/0000-0002-2949-2921 - Adriana Seilicovich

https://orcid.org/0000-0002-0843-6568 - Marianela Candolfi 


\section{Abstract}

Humanin is a mitochondrial-derived peptide which shows robust protective effects against large series of cytotoxic stimuli in many cell types. This makes it an interesting therapeutic target for many diseases, including cancer and neurodegenerative diseases, among others. Furthermore, this peptide could be used as a biomarker for such diseases. Over the last decade, humanin analogs and peptide mimetics have been developed, which exert highly promising results in preclinical models. Besides, the therapeutic potential of gene therapy vectors that overexpress or silence endogenous humanin is under evaluation. Nonetheless, its possible role in cancer progression and chemoresistance are critical issues to be addressed before translating these therapeutic approaches to the clinic. All these matters will be covered in this review.

Keywords: Humanin; mitochondrial-derived peptide; cancer; neurodegenerative diseases.

\section{Introducción}

Desde el descubrimiento de los péptidos mitocondriales, la participación en el metabolismo celular ya no es considerada como la única función de la mitocondria, sino que también se dio importancia a su papel como fuente de factores protectores del estrés, ya sean autócrinos, parácrinos o endócrinos. Estos péptidos son codificados en el genoma mitocondrial y traducidos en la mitocondria o el citoplasma, para seńalizar dentro de la célula o ser liberados y unirse a receptores de membrana. La humanina (HN) fue el primero de estos péptidos mitocondriales en ser descripto (1), y ha demostrado tener efectos citoprotectores robustos en muchos tipos celulares, por lo que es evaluado como un blanco terapéutico potencial para diferentes enfermedades crónicas. Debido a que los péptidos mitocondriales pueden ser detectados en sangre, últimamente han llamado la atención como posibles biomarcadores de diversas enfermedades. En este artículo de revisión, se explorará el papel de la HN como target terapéutico en enfermedades neurodegenerativas y cáncer.

\section{Origen de la $\mathrm{HN}$}

La $\mathrm{HN}$ es un péptido de 24 aminoácidos originalmente aislado de una biblioteca de ADNc mientras se buscaban factores de supervivencia en un área del cerebro no afectada de un paciente con enfermedad de Alzheimer (1). La HN es transcripta a partir de una porción del gen mitocondrial policistrónico MT-RNR2, que codifica al gran ARN ribosomal (ARNr) mitocondrial $16 \mathrm{~S}$ (2). El ADNc de la HN está poliadenilado, por lo que puede estar involucrado en la exportación del transcripto de la HN desde la mitocondria hacia el citoplasma para su posterior traducción. Curiosamente, cuando el ARNm de la HN es traducido den- 
tro de la mitocondria, origina un péptido de 21 aminoácidos. Sin embargo, cuando el mismo se traduce en el citoplasma, resulta en un péptido de 24 aminoácidos. De todas formas, ambos péptidos son funcionales y pueden inhibir la vía apoptótica mitocondrial (3). La HN ha sido detectada tanto en tejidos como en plasma (4-6). Sus niveles circulantes fluctúan acorde a la edad y han sido propuestos como biomarcadores para varias condiciones patológicas.

El marco abierto de lectura (ORF) de 75 bases del ARNr mitocondrial 16S codifica la secuencia aminoacídica Nt-MAPRGFS CLLLLTSEIDLPVKRRA-C $t$ (Nt-Met-Ala-Pro-Arg-Gly-Phe-Ser-CysLeu-Leu-Leu-Leu-Thr-Ser-Glu-Ile-AspLeu-Pro-Val-Lys-Arg-Arg-Ala-Ct), donde GFSCLLLLTSEIDL es la región nuclear hidrofóbica $(3,7)$. Uno de los mecanismos por los cuales actúa la $\mathrm{HN}$ es a través de la unión a su receptor trimérico de membrana (8), por lo que debe ser secretada fuera de la célula después de la traducción para ejercer su función citoprotectora autócrina/ parácrina. La secuencia primaria completa de la HN actúa como una secuencia señal en sí misma, sin necesidad de ser escindida para poder liberarse. La liberación de la HN depende del transporte entre el retículo endoplasmático (RE) y el aparato de Golgi (1). Algunos aminoácidos específicos tienen un papel esencial para la correcta secreción y función de la HN. Estudios de mutagénesis del grupo de Hashimoto et al. (7) revelaron que la $\mathrm{HN}$ tiene una región de 17 aminoácidos que sería la mínima re- gión con máxima actividad citoprotectora, donde Pro, Cys, dos Leu consecutivas, Thr, Ser y Pro son aminoácidos esenciales en las posiciones $3,8,9,12,13,14$ and 19 , respectivamente. La Cys de la posición 8 ha demostrado ser necesaria para la inhibición de la apoptosis pero puede substituirse alternativamente por Lys o Arg (1). Bodzioch y colaboradores postularon que la Pro de la posición 19 permitiría la secreción de la $\mathrm{HN}$, mientras que la unión al receptor sería determinada por Pro, Thr y Ser en las posiciones 3, 13 y 14, respectivamente (9). La estructura secundaria de la HN también depende de ciertos aminoácidos como la Leu y la Ser en las posiciones 9 y 7 , respectivamente, aminoácidos claves si se tiene en cuenta que la inhibición de la formación de dímeros u oligómeros del polipéptido de HN impide su función neuroprotectora (3, 10). Asimismo, la D-Ser en la posición 14 estaría involucrada en la isomerización de la HN (10). También se ha señalado que la $\mathrm{HN}$ adquiere una conformación helicoidal que resulta necesaria para interacciones específicas y su pasaje a través de las membranas (11).

Interesantemente, ORFs adicionales fueron detectados para seis péptidos pequeńos similares a la HN, llamados SHLPs (del inglés small HN-like peptides), en la región mitocondrial del ADN que codifica para la HN (12). SHLP2 y SHLP3, de 26 y 28 aminoácidos respectivamente, inhiben la vía apoptótica intrínseca y mejoran el metabolismo mitocondrial (12). No obstante, aún hay controversia respecto a la fuente 
celular de los péptidos pertenecientes a la familia de la HN, ya que hay evidencia que sugiere que la HN podría también ser codificada desde el ADN genómico, dado que se encontró una homología del 92-95\% con el gen de la $\mathrm{HN}$ en los cromosomas humanos 5, 11 y X (1). Además, se encontraron 12 loci nucleares similares a MT-RNR2 que codifican para 15 péptidos similares a la HN (9). Si bien análisis bioinformáticos detectaron la expresión de 10 genes similares a MT-RNR2 en varios tejidos, incluyendo testículos, riñón, músculo y cerebro, la expresión del gen mitocondrial es considerablemente más alta que la de sus isoformas nucleares en todos los tejidos estudiados (9).

\section{Regulación de la expresión de la HN}

La concentración en plasma de la HN en humanos y ratón, así como en el hipotálamo de rata, va disminuyendo con la edad $(13,14)$. Además, las ratas presentan una pérdida significativa de $\mathrm{HN}$ en el músculo esquelético durante el envejecimiento (14). Debido a estos resultados, se planteó que la $\mathrm{HN}$ podría ser un péptido inhibidor del envejecimiento. De hecho, altos niveles de HN en plasma humano se encuentran asociados a longevidad, como puede observarse en personas centenarias (15). En contraste, la expresión de la HN aumenta en los testículos de rata con el paso del tiempo (16). Ciertas proteínas pueden interactuar con la $\mathrm{HN}$ e inhibir sus efectos neuroprotectores. VSTM2L ( $V$-set and transmembrane domain containing 2 like) puede antagonizar a la $\mathrm{HN}$ tanto en suero como en el sistema nervioso central (SNC) (17), así como los niveles de HN circulante pueden controlarse por su interacción con el factor de crecimiento similar a la insulina (IGF) y proteínas de unión a IGF (IGFBP). IGFBP-3 es una proteína transportadora de IGF-I y HN que se une con alta afinidad a la $\mathrm{HN}$, lo cual conlleva a la inhibición de la muerte celular mediada por IGFBP-3 (10) mientras también regula los niveles circulantes de $\mathrm{HN}$. Aparentemente, IGFBP-3 también podría regular la distribución específica de la $\mathrm{HN}$ en los tejidos, así como su acción (18). Por ejemplo, la HN generada en tejidos periféricos puede trasportarse por IGFBP-3 al SNC (19). Por otro lado, modelos de ratones transgénicos que expresan hormona de crecimiento $(\mathrm{GH})$ tienen altos niveles de IGF-I y, a su vez, bajos niveles de $\mathrm{HN}$ en plasma (20). Por el contrario, ratones enanos Ames (Prop-1DF) con mutaciones en el gen Prop-1 tienen niveles plasmáticos elevados de $\mathrm{HN}$, mientras que los niveles de GH y IGF-I son indetectables (20).

Los mecanismos de regulación de la biosíntesis de la HN aún no han sido esclarecidos por completo. Resultados previos de nuestro grupo indican que la expresión de la HN en la glándula pituitaria anterior presenta dimorfismo sexual, siendo más baja en células pituitarias de hembras que de machos, y es directamente inhibida por estrógenos (21). Ha sido observado que la expresión de $\mathrm{HN}$ es regulada positivamente por inductores de estrés mitocondrial, tales como la deprivación de suero y drogas 
quimioterapéuticas $(9,22,23)$, mientras que es regulada negativamente por factores antiapoptóticos como el $\beta$-caroteno (9). Respecto a la degradación y el reciclaje de la HN, TRIM11 (motivo tripartito 11) es una de las proteínas propuestas como reguladora de los niveles intracelulares de $\mathrm{HN}$, dirigiendo su degradación por el proteasoma (19).

\section{Mecanismos de acción de la HN}

La HN puede ejercer su acción citoprotectora por medio de la regulación de la vía apoptótica intrínseca o mitocondrial, modulando la expresión y localización intracelular de las proteínas de la familia $\mathrm{Bcl}-2$. Un trabajo reciente de nuestro grupo muestra que el silenciamiento de la $\mathrm{HN}$, utilizando vectores baculovirales que codifican ARNsh específicos, regula positivamente la expresión de Bax (24). Esto sugiere que, en condiciones basales, la $\mathrm{HN}$ mantiene inhibida la expresión de Bax (24). La HN puede interactuar con las proteínas proapoptóticas Bax, Bid y su isoforma truncada tBid en el citosol, evitando su translocación a la mitocondria e inhibiendo la liberación del citocromo c, la formación del apoptosoma y la activación de la caspasa $3(22,25)$. La HN no puede eliminar la unión de Bax y tBid a la membrana mitocondrial, pero su interacción dificulta el reclutamiento del Bax citosólico y su oligomerización en la membrana, lo cual inhibe la formación de poros y los eventos subsiguientes que desencadenan la vía apoptótica intrínseca (26). Además, la $\mathrm{HN}$ y sus análogos reducen la expresión de Bax mientras incrementan la de Bcl-2 en células endoteliales (27). Recientemente, pudimos demostrar que la HN exógena regula positivamente la expresión de $\mathrm{Bcl}-2$ en células pituitarias, disminuyendo el radio Bax/Bcl-2 e impidiendo, una vez más, la translocación de Bax a la mitocondria (28). También fue observado que la HN exógena puede ser incorporada por células del epitelio pigmentario de la retina (RPE) y relocalizarse en la mitocondria, impidiendo de esta manera la formación de especies reactivas de oxígeno, restaurando la bioenergética mitocondrial e inhibiendo la senescencia y muerte celular (29).

La HN también puede liberarse de la célula, unirse a receptores de membrana y tener efectos autócrinos, parácrinos o endócrinos. Uno de los receptores de membrana para HN está compuesto por tres subunidades: CNTFR, WSX-1 y gp130 de 130 kDa (8, 30). La unión de la HN induce heterodimerización de los dominios intracelulares de WSX-1 y gp130 (31). Luego de unirse al receptor, la $\mathrm{HN}$ puede activar muchas cascadas de señalización, tales como RAS/ MAPKs, PI3K, JNK y STAT3 $(32,33)$. La trimerización del receptor induce la activación de JAK2 quinasa, seguida de la activación de STAT3 (33). Los STATs dimerizados se trasladan al núcleo y regulan la transcripción génica. Además, las tirosina quinasas AKT y p38 han sido involucradas en la actividad neuroprotectora de la $\mathrm{HN}$ $(33,34)$. Otro mecanismo involucrado en la acción de la HN es la activación del receptor péptido formil símil (FPRL-1), que 
induce la activación de ERK1/2 (30, 35). La HN también ejerce su función citoprotectora por medio de la activación de la vía AMPK-eNOS (5). Nuestro grupo demostró recientemente que la vía de NF-KB está también involucrada en la acción antiapoptótica de la HN (28). Llamativamente, la translocación nuclear de NF-KB-p65 inducida por $\mathrm{HN}$ sólo se observó en células pituitarias tumorales pero no en células pituitarias normales (28). Otro mecanismo neuroprotector de la $\mathrm{HN}$ comprende a la familia de proteínas inhibidoras apoptóticas (IAPs) tras la activación de la vía JAK/ STAT3. La HN regula positivamente la expresión de Apollon/Bruce, que se une a Smac/Diablo y la caspasa 9, lo que conlleva a la degradación de estas proteínas y la inhibición de la apoptosis (36).

La autofagia es otro de los mecanismos que dispara la HN. Ésta aumenta la expresión de LC3-II, un marcador del autofagosoma, y el número de autofagosomas y autolisosomas (37). Adicionalmente, la HN se localiza en la membrana lisosomal, donde coopera para mejorar la eficiencia de la autofagia mediada por chaperonas (37). La HN puede también estabilizar la unión de la chaperona HSP90 a sus sustratos en la cara citosólica de la membrana lisosomal (37).

\section{HN y neurodegeneración}

La enfermedad de Alzheimer (EA) es la principal causa de demencia. Los eventos moleculares patogénicos que causan esta enfermedad tienen la participación del pép- tido $\beta$-amiloide $(A \beta)$ y la proteína tau, los componentes más importantes de placas y ovillos neurofibrilares, respectivamente (38). Desde que Nishimito y su grupo descubrieron la $\mathrm{HN}$ en el lóbulo occipital de pacientes con EA, este péptido ha demostrado tener un gran potencial para el tratamiento de enfermedades neurodegenerativas, dado que puede proteger a las neuronas de los agravios ocasionados por genes relacionados a la EA y la toxicidad por $A \beta$ (39). La primera evidencia in vivo de este efecto neuroprotector fue informada por Mamiya et al., quienes administraron un análogo potente de la HN, S14G-HN (HNG), que mejoró notablemente los déficits en aprendizaje y memoria ocasionados por la escopolamina en ratones (40). Más tarde, la administración del análogo HNG a ratones triple transgénicos con mutaciones en APPswe, tauP310L y PS-1M146V, asemejándose al cuadro de la EA, pudo reducir la acumulación de $A \beta$ y mejorar la función cognitiva en la etapa temprana de acumulación de placas (41). Asimismo, la eficacia de HNG ha sido evaluada en modelos preclínicos con placas amiloides preexistentes bien establecidas, usando un modelo doble transgénico de EA (ratones portadores de los genes humanos mutantes APPswe y PS1-dE9). En concordancia con el trabajo previo, el tratamiento mejoró el déficit cognitivo y permitió la recuperación de la función de memoria espacial. En este estudio también se sugiere que HNG podría reducir la neuroinflamación asociada a $A \beta$ (42). Otros trabajos demuestran que la administración de HN puede disminuir la 
producción de citoquinas proinflamatorias, tales como IL-6, IL-1 $\beta$ y TNF-a, inducida en astrocitos por LPS (43) y en un modelo murino de hemorragia cerebral (44). En otro estudio, se observó que la inyección intra-hipocampo de HNG protege a ratas de los déficits inducidos por $A \beta$ en la memoria espacial a largo plazo, por un mecanismo que comprende el bloqueo de la activación de la caspasa 3 inducida por $A \beta$ y la supresión de STAT3 (45).

Chiba et al. desarrollaron un péptido híbrido, colivelin, que está compuesto por el factor neurotrófico dependiente de actividad (ADNF) fusionado a un análogo de la $\mathrm{HN}$, AGA-(C8R)HNG17. Este péptido demostró su efecto neuroprotector al ser inyectado en muy bajas dosis por vía intracerebroventricular en modelos de EA (46). Esto se debió tanto a la activación de la proteína quinasa IV dependiente de $\mathrm{Ca}^{2+} /$ calmodulina por ADNF, como a la activación de STAT3 por HNG17 (46), que resultaban en un efecto citoprotector aún más fuerte que el ocasionado por la administración simultánea de HNG17 y ADNF (47).

La EA no es la única patología neurológica que podría beneficiarse del potencial efecto terapéutico de la HN. La excitotoxicidad del receptor $\mathrm{N}$-metil-D-aspartato (NMDA) se encontró tanto en la EA como en la enfermedad de Parkinson. Nuevos estudios muestran que la exposición a NMDA disminuye la viabilidad de las neuronas corticales de rata y que, a su vez, la incubación de estas células con HN atenúa la disfun- ción mitocondrial generada (48). Estos resultados han fomentado el desarrollo de un péptido mimético que se une a gp130 en los mismos sitios de unión, imitando los efectos citoprotectores de la $\mathrm{HN}$ en neuronas (49).

Estrategias terapéuticas similares podrían aplicarse para el tratamiento de isquemia/ reperfusión en derrames cerebrales. Utilizando un modelo in vitro de deprivación de oxígeno y glucosa alternada con reoxigenación en una línea celular humana derivada de un neuroblastoma, llamada SH-SY5Y, la HN demostró ser un buen inhibidor de la apoptosis, tras la reactivación de la señalización JAK2/STAT3 (50). Un trabajo reciente muestra un nuevo papel de la $\mathrm{HN}$ en un modelo murino de oclusión de arteria media, donde HNG pudo atenuar el infarto cerebral, así como proteger a las células endoteliales cerebrales de la deprivación de oxígeno, posiblemente por un mecanismo que incluye a gp130, PI3K/AKT y la vía de NF-KB (44). No obstante, aún se desconoce si la HN cruza la barrera hemato-encefálica por medio de transportadores o por receptores de $\mathrm{HN}$ en la superficie de las células endoteliales.

Otra condición progresiva es la degeneración macular asociada a la edad (AMD), que causa la pérdida irreversible de la visión central y carece de tratamiento en el 90\% de los pacientes (51). Esta patología se caracteriza por una etapa temprana en la que agregados extracelulares insolubles se acumulan en la retina, seguida de una etapa 
tardía en la que se producen áreas de degeneración células RPE, que sirven de apoyo para los fotorreceptores de la retina (51). El grupo de Hinton demostró que las células RPE pueden expresar HN, así como también tomar $\mathrm{HN}$ exógena del medio, lo cual sugiere que la $\mathrm{HN}$ podría proteger a estas células del estrés oxidativo provocado por $\mathrm{H}_{2} \mathrm{O}_{2}$, efecto mediado por el incremento de la bioenergética y biogénesis mitocondrial (29). Luego observaron que durante el desarrollo de AMD, estresores del RE pueden inducir la muerte de células RPE aumentando la producción mitocondrial de superóxido. De acuerdo con los resultados anteriores, la HN protegió eficientemente a las células RPE de la apoptosis, restaurando el glutatión mitocondrial (52).

\section{HN y cáncer}

El equilibrio entre la proliferación y la apoptosis mantiene la homeostasis de los tejidos. Las células tumorales generalmente mantienen su supervivencia suprimiendo la apoptosis, por lo que alteraciones en la tasa apoptótica tienen consecuencias en el desarrollo del tumor. El efecto antiapoptótico de la HN aumenta la probabilidad de que este péptido posea un papel en la patogénesis del cáncer. Si bien la HN fue propuesta como un posible oncopéptido en el 2002 (53), continúa siendo escaso el número de trabajos que involucran a la $\mathrm{HN}$ en el desarrollo tumoral. Su expresión fue detectada en cáncer gástrico, por lo que fue propuesto que la sobreexpresión de este péptido podría constituir un evento molecular impor- tante de la tumorigénesis y la quimiorresistencia del tumor (54). Más recientemente, también se informó su sobreexpresión en células tumorales de vejiga, por lo que se sugirió a la HN circulante en sangre como un potencial biomarcador para el diagnóstico de este tipo de cáncer(55).

La mayoría de las drogas quimioterapéuticas actúan disminuyendo la tasa de proliferación y/o aumentando la de apoptosis. Aunque el papel de la HN en la respuesta tumoral a las drogas citotóxicas es controvertido, este péptido mostraría un efecto citoprotector en células normales expuestas a quimioterapéuticos $(56,57)$. El análogo HNG revirtió el arresto del ciclo celular en hueso inducido por la quimioterapia con bortezomib, un inhibidor del proteasoma, sin interferir en el efecto anticancerígeno de la droga, tanto en xenoinjertos de meduloblastoma y neuroblastoma, como en líneas celulares humanas (58). Asimismo, en un modelo murino de melanoma metastásico de pulmón se observó que HNG protegía contra la pérdida de leucocitos y células germinales masculinas inducida por ciclofosfamida y, a su vez, incrementaba la supresión de formación de metástasis generada por la ciclofosfamida $(56,57)$. Es por ello que fue propuesto que análogos de la HN podrían ser buenos adyuvantes para la quimioterapia, al reducir la inducción de leucopenia y el bajo conteo de espermatozoides $(56,57,59)$. De hecho, los análogos de HN podrían incrementar la respuesta tumoral al bortezomib (58) y la ciclofosfamida (56). Sin embargo, resultados recien- 
tes de nuestro laboratorio contradicen los hallazgos antes mencionados. La inhibición de la HN endógena por inyección intratumoral de un vector baculoviral que codifica un ARNsh específico de $\mathrm{HN}$, reguló positivamente la expresión de Bax, incrementó la tasa apoptótica tumoral, inhibió el crecimiento tumoral y aumentó la supervivencia de modelos con xenoinjertos de prolactinoma (24). Además, nuestros resultados preliminares indican que la administración sistémica de $\mathrm{HN}$ favorece la progresión tumoral y la quimiorresistencia en modelos murinos de cáncer de mama (60), lo cual cuestiona la seguridad de la administración sistémica de HN o sus análogos para el tratamiento del cáncer.

Hemos mostramos previamente que la expresión de $\mathrm{HN}$ es más elevada en células de prolactinoma que en células pituitarias normales, en las cuales los estrógenos inhiben la expresión de $\mathrm{HN}$, una vía regulatoria que se encuentra suprimida en células tumorales $(21,28)$. Como se describió anteriormente, la unión de la $\mathrm{HN}$ a su receptor trimérico CNTFR/gp130/WSX-1 activa la señalización de ERK1/2, AKT y STAT3 (34). NF-KB y STAT3 son fundamentales para el desarrollo de muchos tipos de tumores tales como el glioblastoma o el cáncer de mama triple negativo, en los cuales estos mediadores están sobreexpresados e hiperactivados (61-63). De hecho, nuestro grupo demostró que el efecto citoprotector de la $\mathrm{HN}$ en células tumorales pituitarias involucra a las vías de NF-KB y STAT3 (21, $28)$. En concordancia, en un estudio se ob- servó que la inhibición de la señalización de gp130 y de la fosforilación de AKT, ERK y STAT3, mejoró la actividad antitumoral de un agente quimioterapéutico para cáncer de colon (64). Además, dosis elevadas de $\mathrm{HN}$ disminuyeron la apoptosis inducida por el ER en células de glioma incubadas con tunicamicina, un inhibidor de la glicosilación (52).

También fue demostrado que los péptidos Bax y tBid están involucrados en el efecto antiapoptótico de $\mathrm{HN}$ en células tumorales, pituitarias (28) o de glioblastoma, en las cuales la inhibición de la HN endógena mediado por ARNsi, sensibilizó a las células a la apoptosis causada por estaurosporina y deprivación de suero (22), sugiriendo que la inhibición de la vía apoptótica mitocondrial a través de la regulación positiva de HN podría contribuir a la patogénesis tumoral. También fue observado un efecto protector de SHLP2 y SHLP3 en células de cáncer prostático, en las cuales inhibieron la apoptosis por deprivación de suero y restauraron el metabolismo mitocondrial (12). Los niveles circulantes de SHLP2 resultaron estar negativamente correlacionados con el riesgo de aparición de cáncer de próstata, un hecho que fue asociado a la disfunción mitocondrial durante la carcinogénesis (65). Esto podría reflejarse en cambios en los patrones de secreción de péptidos mitocondriales en células cancerosas.

La senescencia celular tendría tanto efectos benéficos como perjudiciales dependiendo del contexto (66), teniendo un papel con- 
trovertido en el desarrollo del cáncer (67). Entre las desventajas, el fenotipo secretor asociado a la senescencia (SASP) puede perturbar el microambiente, alterar la estructura y el funcionamiento de los tejidos normales, y promover fenotipos malignos en células cercanas. Por lo tanto, la respuesta inflamatoria de la senescencia favorecería la proliferación e invasión tumoral (68, 69). Cuando las células senescentes no son eliminadas por el sistema inmune, el SASP puede generar inflamación crónica e incrementar el riesgo de incidencia de cáncer (70). Muy recientemente, fue demostrado que en fibroblastos dérmicos humanos que presentaban senescencia inducida por doxorrubicina aumentó la expresión de $\mathrm{HN}$, que a su vez incrementó la respiración mitocondrial y la producción de factores de SASP (66). Estos resultados sugieren que el bloqueo de esta respuesta podría ser una buena estrategia terapéutica para el tratamiento del cáncer.

\section{Discusión}

El efecto citoprotector de la $\mathrm{HN}$ ha conducido al desarrollo de análogos de HN para el tratamiento de enfermedades crónicas. La relación directa entre secuencia y función de la molécula de HN permite el desarrollo de análogos con propiedades agonistas y antagonistas específicas, mediante substituciones de aminoácidos particulares. Entre todos los análogos producidos se encuentran HNG, HNGF6A y colivelin, los cuales mejoran la actividad citoprotectora de la HN endógena contra insultos neuro- tóxicos $(10,71)$, aumentan su disponibilidad en sangre y su vida media (71) o tienen fuertes efectos neuroprotectores bajo un amplio rango de concentraciones $(46,72)$, respectivamente. Considerando la eficacia de los análogos administrados en modelos preclínicos de enfermedades degenerativas, se propuso su administración sistémica para el tratamiento de $\mathrm{AD}$ o arterosclerosis, entre otras, o para proteger la fertilidad de pacientes con cáncer recibiendo quimioterapia. Sin embargo, aún hay controversia respecto a la biodistribución de estos péptidos. Si bien análogos potentes como colivelin ejercen neuroprotección en modelos preclínicos tras su administración i.p. o i.v., sólo una pequeña fracción de los mismos ingresa al cerebro (46). Estudios de biodistribución indican que los análogos de la HN no se concentran efectivamente en los órganos blanco, como el cerebro, mediante la administración i.p. (71). De hecho, estos péptidos carecen de especificidad y pueden actuar en receptores de $\mathrm{HN}$ expresados ubicuamente cuando concentraciones elevadas alcanzan la corriente sanguínea, luego de su administración sistémica. Considerando que los desórdenes neurodegenerativos requieren administración crónica de los agentes citoprotectores, deben evaluarse métodos no invasivos que alcancen concentraciones terapéuticas de estos análogos en el cerebro. La administración intranasal de los péptidos podría superar las restricciones de la barrera hemato-encefálica de manera mucho más eficiente que la administración sistémica (73). La administración nasal de colivelin durante 3 semanas mejoró los pro- 
blemas de memoria en modelos murinos de EA y fue propuesta como una estrategia alternativa para que los péptidos puedan acceder al cerebro (74). La nanotecnología también podría mejorar la biodistribución. La odorranalectina, un péptido pequeño no inmunogénico con comportamiento similar a la lectina, facilitó el acceso al cerebro de HNG transportado con nanopartículas tras la administración intranasal y aumentó su efecto terapéutico en modelos murinos de EA (75). Por otro lado, la expresión local de HN o sus análogos mediante vectores de terapia génica podría aumentar su disponibilidad y dirigir su expresión a órganos específicos. Utilizar promotores específicos permitiría una regulación ajustada de la expresión, en caso de aparición de efectos adversos.

A pesar de que la base de datos del GenBank indica que el 99,4\% de las secuencias humanas de HN no están afectadas por mutaciones (76), este péptido parece constituir un importante blanco terapéutico para el tratamiento del cáncer. Resultados preliminares de nuestro laboratorio indican que la administración sistémica de la HN facilita la progresión tumoral y la quimiorresistencia en modelos murinos de cáncer de mama (60). Si bien estos descubrimientos se contradicen con informes de otros grupos donde describen una mejora de la quimiosensibilidad en modelos preclínicos de cáncer tratados con análogos de la HN (56, 58), en nuestro trabajo reciente reportamos que el tratamiento con vectores de terapia génica que silencian la expresión de $\mathrm{HN}$ en- dógena, inhibe el crecimiento tumoral en un modelo murino de prolactinoma (24). En concordancia con nuestros resultados, se observó que el silenciamiento de la HN reduce la supervivencia de células de glioblastoma (22), en las que sus mediadores ERK2 y STAT3 son regulados positivamente. Será necesario resolver esta controversia con más estudios antes de la traslación de los análogos de la HN a la práctica clínica.

\section{Conclusión}

Pese a que las estrategias terapéuticas enfocadas a la $\mathrm{HN}$ aún requieren una investigación más profunda antes de ser trasladadas a la clínica, estos enfoques tienen un gran potencial para el tratamiento del cáncer y enfermedades neurodegenerativas.

\section{Financiamiento}

Este trabajo fue financiado por el Consejo Nacional de Investigaciones Científicas y Tecnológicas (beca doctoral otorgada a AS Asad y beca postdoctoral otorgada a MF Gottardo), Agencia Nacional de Promoción Científica y Tecnológica (PICT 2015-3309 de M Candolfi; PICT 2014-0334 de A Seilicovich; beca doctoral de AJ Nicola Candia y CF Zuccato), Instituto Nacional del Cáncer (Argentina, Asistencia Financiera IV a M Candolfi) y la Universidad Buenos Aires. 


\section{Referencias}

1. Hashimoto Y, Niikura T, Tajima H, Yasukawa T, Sudo H, Ito Y, et al. A Rescue Factor Abolishing Neuronal Cell Death by a Wide Spectrum of Familial Alzheimer's Disease Genes and Abeta. Proc Natl Acad Sci U S A. 2001; 98(11): p. 6336-41.

2. Lee C, Yen K, and Cohen P. Humanin: A Harbinger of Mitochondrial-Derived Peptides? Trends in endocrinology and metabolism: TEM. 2013; 24(5): p. 222-8.

3. Yamagishi Y, Hashimoto Y, Niikura T, and Nishimoto I. Identification of Essential Amino Acids in Humanin, a Neuroprotective Factor against Alzheimer's Disease-Relevant Insults. Peptides. 2003; 24(4): p. 585-95.

4. Charununtakorn ST, Shinlapawittayatorn K, Chattipakorn SC, and Chattipakorn N. Potential Roles of Humanin on Apoptosis in the Heart. Cardiovascular Therapeutics. 2016; 34(2): p. 107-14.

5. Muzumdar RH, Huffman DM, Calvert JW, Jha S, Weinberg Y, Cui L, et al. Acute Humanin Therapy Attenuates Myocardial Ischemia and Reperfusion Injury in Mice. Arteriosclerosis, Thrombosis, and Vascular Biology. 2010; 30(10): p. 1940-8.

6. Tajima H, Niikura T, Hashimoto Y, Ito Y, Kita Y, Terashita K, et al. Evidence for in Vivo Production of Humanin Peptide, a Neuroprotective Factor against Alzheimer's Disease-Related Insults. Neurosci Lett. 2002; 324(3): p. 227-31.

7. Hashimoto $Y$, Niikura T, Ito $Y$, Sudo H, Hata M, Arakawa E, et al. Detailed Characterization of Neuroprotection by a Rescue Factor Humanin against Various Alzheimer's Disease-Relevant Insults. J Neurosci. 2001; 21(23): p. 9235-45.

8. Hashimoto Y, Kurita M, Aiso S, Nishimoto I, and Matsuoka M. Humanin Inhibits Neuronal Cell Death by Interacting with a Cytokine Receptor Complex or Complexes Involving Cntf Receptor Alpha/Wsx-1/Gp130. Mol Biol Cell. 2009; 20(12): p. 2864-73.
9. Bodzioch $\mathrm{M}$, Lapicka-Bodzioch $\mathrm{K}$, Zapala B, Kamysz W, Kiec-Wilk B, and Dembinska-Kiec A. Evidence for Potential Functionality of Nuclearly-Encoded Humanin Isoforms. Genomics. 2009; 94(4): p. 247-56.

10. Terashita K, Hashimoto Y, Niikura T, Tajima H, Yamagishi $\mathrm{Y}$, Ishizaka $\mathrm{M}$, et al. Two Serine Residues Distinctly Regulate the Rescue Function of Humanin, an Inhibiting Factor of Alzheimer's Disease-Related Neurotoxicity: Functional Potentiation by Isomerization and Dimerization. Journal of Neurochemistry. 2003; 85(6): p. 1521-38.

11. Benaki D, Zikos C, Evangelou A, Livaniou E, Vlassi M, Mikros E, et al. Solution Structure of Humanin, a Peptide against Alzheimer's Disease-Related Neurotoxicity. Biochem Biophys Res Commun. 2005; 329(1): p. 152-60.

12. Cobb LJ, Lee C, Xiao J, Yen K, Wong RG, Nakamura HK, et al. Naturally Occurring Mitochondrial-Derived Peptides Are Age-Dependent Regulators of Apoptosis, Insulin Sensitivity, and Inflammatory Markers. Aging. 2016; 8(4): p. 796-809.

13. Bachar AR, Scheffer L, Schroeder AS, Nakamura HK, Cobb LJ, Oh YK, et al. Humanin Is Expressed in Human Vascular Walls and Has a Cytoprotective Effect against Oxidized Ldl-Induced Oxidative Stress. Cardiovascular Research. 2010; 88(2): p. 360-6.

14. Muzumdar RH, Huffman DM, Atzmon G, Buettner C, Cobb LJ, Fishman S, et al. Humanin: A Novel Central Regulator of Peripheral Insulin Action. PloS one. 2009; 4(7): p. e6334.

15. Conte M, Ostan R, Fabbri C, Santoro A, Guidarelli G, Vitale G, et al. Human Aging and Longevity Are Characterized by High Levels of Mitokines. The Journals of Gerontology. Series A, Biological Sciences and Medical Sciences. 2018; 74(5): p. 600-7.

16. Gong Z, Tas E, and Muzumdar R. Humanin and Age-Related Diseases: A New Link? Frontiers in Endocrinology. 2014; 5: p. 210. 
17. Rossini L, Hashimoto Y, Suzuki H, Kurita M, Gianfriddo M, Scali C, et al. Vstm21 Is a Novel Secreted Antagonist of the Neuroprotective Peptide Humanin. FASEB Journal : Official Publication of the Federation of American Societies for Experimental Biology. 2011; 25(6): p. 1983-2000.

18. Xiao J, Kim SJ, Cohen P, and Yen K. Humanin: Functional Interfaces with Igf-I. Growth hormone \& IGF research : official journal of the Growth Hormone Research Society and the International IGF Research Society. 2016; 29: p. 21-27.

19. Niikura T, Hashimoto Y, Tajima H, Ishizaka M, Yamagishi Y, Kawasumi M, et al. A Tripartite Motif Protein Trim11 Binds and Destabilizes Humanin, a Neuroprotective Peptide against Alzheimer's Disease-Relevant Insults. The European Journal of Neuroscience. 2003; 17(6): p. 1150-8.

20. Lee C, Wan J, Miyazaki B, Fang Y, Guevara-Aguirre J, Yen K, et al. Igf-I Regulates the Age-Dependent Signaling Peptide Humanin. Aging Cell. 2014; 13(5): p. 958-61.

21. Gottardo MF, Jaita G, Magri ML, Zarate S, Moreno Ayala M, Ferraris J, et al. Antiapoptotic Factor $\mathrm{Hu}-$ manin Is Expressed in Normal and Tumoral Pituitary Cells and Protects Them from Tnf-Alpha-Induced Apoptosis. PloS one. 2014; 9(10): p. e111548.

22. Guo B, Zhai D, Cabezas E, Welsh K, Nouraini S, Satterthwait AC, et al. Humanin Peptide Suppresses Apoptosis by Interfering with Bax Activation. Nature. 2003 ; 423(6938): p. 456-61.

23. Jia Y, Lue YH, Swerdloff R, Lee KW, Cobb LJ, Cohen $\mathrm{P}$, et al. The Cytoprotective Peptide Humanin Is Induced and Neutralizes Bax after Pro-Apoptotic Stress in the Rat Testis. Andrology. 2013; 1(4): p. 651-9.

24. Gottardo MF, Pidre ML, Zuccato C, Asad AS, Imsen M, Jaita G, et al. Baculovirus-Based Gene Silencing of Humanin for the Treatment of Pituitary Tumors. Apoptosis : An International Journal on Programmed Cell Death. 2018; 23(2): p. 143-151.
25. Zhai D, Luciano F, Zhu X, Guo B, Satterthwait AC, and Reed JC. Humanin Binds and Nullifies Bid Activity by Blocking Its Activation of Bax and Bak. The Journal of Biological Chemistry. 2005; 280(16): p. 15815-24.

26. Ma ZW and Liu DX. Humanin Decreases Mitochondrial Membrane Permeability by Inhibiting the Membrane Association and Oligomerization of Bax and Bid Proteins. Acta Pharmacologica Sinica. 2018; 39(6): p. 1012-1021.

27. Xie Y, Liu ZH, Li XY, Zhou YD, Xu X, Hu LF, et al. Protection Effect of [Gly14]-Humanin from Apoptosis Induced by High Glucose in Human Umbilical Vein Endothelial Cells. Diabetes Research and Clinical Practice. 2014; 106(3): p. 560-6.

28. Gottardo MF, Moreno Ayala M, Ferraris J, Zarate $S$, Pisera D, Candolfi $M$, et al. Humanin Inhibits Apoptosis in Pituitary Tumor Cells through Several Signaling Pathways Including Nf-Kappab Activation. Journal of Cell Communication and Signaling. 2017; 11(4): p. 329-340.

29. Sreekumar PG, Ishikawa K, Spee C, Mehta HH, Wan J, Yen K, et al. The Mitochondrial-Derived Peptide Humanin Protects Rpe Cells from Oxidative Stress, Senescence, and Mitochondrial Dysfunction. Investigative Ophthalmology \& Visual Science. 2016; 57(3): p. 1238-53.

30. Matsuoka M and Hashimoto Y. Humanin and the Receptors for Humanin. Molecular Neurobiology. 2010; 41(1): p. 22-8.

31. Hashimoto Y, Kurita M, and Matsuoka M. Identification of Soluble Wsx-1 Not as a Dominant-Negative but as an Alternative Functional Subunit of a Receptor for an Anti-Alzheimer's Disease Rescue Factor Humanin. Biochemical and Biophysical Research Communications. 2009; 389(1): p. 95-9.

32. Hoang PT, Park P, Cobb LJ, Paharkova-Vatchkova V, Hakimi M, Cohen P, et al. The Neurosurvival Factor Humanin Inhibits Beta-Cell Apoptosis Via Signal Transducer and Activator of Transcription 3 Activation and Delays and Ameliorates Diabetes in 
Nonobese Diabetic Mice. Metabolism: Clinical and Experimental. 2010; 59(3): p. 343-9.

33. Hashimoto Y, Suzuki H, Aiso S, Niikura T, Nishimoto I, and Matsuoka M. Involvement of Tyrosine Kinases and Stat 3 in Humanin-Mediated Neuroprotection. Life Sciences. 2005; 77(24): p. 3092-104.

34. Kim SJ, Guerrero N, Wassef G, Xiao J, Mehta HH, Cohen P, et al. The Mitochondrial-Derived Peptide Humanin Activates the Erk1/2, Akt, and Stat3 Signaling Pathways and Has Age-Dependent Signaling Differences in the Hippocampus. Oncotarget. 2016; 7(30): p. 46899-46912.

35. Ying G, Iribarren P, Zhou Y, Gong W, Zhang N, Yu ZX, et al. Humanin, a Newly Identified Neuroprotective Factor, Uses the G Protein-Coupled Formylpeptide Receptor-Like-1 as a Functional Receptor. J Immunol. 2004; 172(11): p. 7078-85.

36. Hashimoto $Y$, Takeshita $Y$, Naito M, Uchino H, and Matsuoka M. Apollon/Bruce Is Upregulated by Humanin. Molecular and cellular biochemistry. 2014; 397(1-2): p. 147-55.

37. Gong $Z$ and Tasset I. Humanin Enhances the Cellular Response to Stress by Activation of Chaperone-Mediated Autophagy. Oncotarget. 2018; 9(13): p. 10832-10833.

38. Scheltens P, Blennow K, Breteler MM, de Strooper B, Frisoni GB, Salloway S, et al. Alzheimer's Disease. Lancet. 2016; 388(10043): p. 505-17.

39. Hashimoto Y, Ito Y, Niikura T, Shao Z, Hata M, Oyama F, et al. Mechanisms of Neuroprotection by a Novel Rescue Factor Humanin from Swedish Mutant Amyloid Precursor Protein. Biochemical and Biophysical Research Communications. 2001; 283(2): p. 460-8.

40. Mamiya T and Ukai M. [Gly(14)]-Humanin Improved the Learning and Memory Impairment Induced by Scopolamine in Vivo. Br J Pharmacol. 2001; 134(8): p. 1597-9.

41. Niikura T, Sidahmed E, Hirata-Fukae C, Aisen PS, and Matsuoka Y. A Humanin Derivative Reduces
Amyloid Beta Accumulation and Ameliorates Memory Deficit in Triple Transgenic Mice. PloS one. 2011; 6(1): p. e16259.

42. Zhang W, Li Z, Hao J, Zhang Z, Liu L, Mao N, et al. S14g-Humanin Improves Cognitive Deficits and Reduces Amyloid Pathology in the Middle-Aged Appswe/Ps1de9 Mice. Pharmacol Biochem Behav. 2012; 100(3): p. 361-9.

43. Zhao ST, Zhao L, and Li JH. Neuroprotective Peptide Humanin Inhibits Inflammatory Response in Astrocytes Induced by Lipopolysaccharide. Neurochemical Research. 2013; 38(3): p. 581-8.

44. Peng T, Wan W, Wang J, Liu Y, Fu Z, Ma X, et al. The Neurovascular Protective Effect of S14g-Humanin in a Murine Mcao Model and Brain Endothelial Cells. IUBMB Life. 2018; 70(7): p. 691-699.

45. Yuan L, Liu XJ, Han WN, Li QS, Wang ZJ, Wu MN, et al. [Gly14]-Humanin Protects against Amyloid Beta Peptide-Induced Impairment of Spatial Learning and Memory in Rats. Neuroscience Bulletin. 2016; 32(4): p. 374-82.

46. Chiba T, Yamada M, Hashimoto Y, Sato M, Sasabe J, Kita Y, et al. Development of a Femtomolar-Acting Humanin Derivative Named Colivelin by Attaching Activity-Dependent Neurotrophic Factor to Its N Terminus: Characterization of Colivelin-Mediated Neuroprotection against Alzheimer's Disease-Relevant Insults in Vitro and in Vivo. J Neurosci. 2005; 25(44): p. 10252-61.

47. Yin R, Yin K, Guo Z, Zhang Z, Chen L, Cao L, et al. Protective Effects of Colivelin against Alzheimer's Disease in a Pdapp Mouse Model. Cell Physiol Biochem. 2016; 38(3): p. 1138-46.

48. Cui AL, Zhang YH, Li JZ, Song T, Liu XM, Wang $\mathrm{H}$, et al. Humanin Rescues Cultured Rat Cortical Neurons from Nmda-Induced Toxicity through the Alleviation of Mitochondrial Dysfunction. Drug Design, Development and Therapy. 2017; 11: p. 1243-1253.

49. Alam MP, Bilousova T, Spilman P, Vadivel K, Bai 
D, Elias CJ, et al. A Small Molecule Mimetic of the Humanin Peptide as a Candidate for Modulating Nmda-Induced Neurotoxicity. ACS Chemical Neuroscience. 2018; 9(3): p. 462-468.

50. Gao GS, Li Y, Zhai H, Bi JW, Zhang FS, Zhang $\mathrm{XY}$, et al. Humanin Analogue, S14g-Humanin, Has Neuroprotective Effects against Oxygen Glucose Deprivation/Reoxygenation by Reactivating Jak2/ Stat3 Signaling through the Pi3k/Akt Pathway. Experimental and Therapeutic Medicine. 2017; 14(4): p. 3926-3934.

51. Ambati J and Fowler BJ. Mechanisms of Age-Related Macular Degeneration. Neuron. 2012; 75(1): p. 26-39.

52. Garzón P, Sandra Johanna et al. Características cognitivas y oculares en enfermedad de Alzheimer. Nova, [S.1.], v. 16, n. 29, p. 101 - 114, sep. 2018. ISSN 2462-9448. Disponible en: <http:// hemeroteca.unad.edu.co/index.php/nova/article/ view/2693>. Fecha de acceso: 14 mar. 2019 doi:https://doi.org/10.22490/24629448.2693.

53. Matsunaga D, Sreekumar PG, Ishikawa K, Terasaki H, Barron E, Cohen P, et al. Humanin Protects Rpe Cells from Endoplasmic Reticulum Stress-Induced Apoptosis by Upregulation of Mitochondrial Glutathione. PLoS One. 2016; 11(10): p. e0165150.

54. Maximov V, Martynenko A, Hunsmann G, and Tarantul V. Mitochondrial 16s Rrna Gene Encodes a Functional Peptide, a Potential Drug for Alzheimer's Disease and Target for Cancer Therapy. Medical Hypotheses. 2002; 59(6): p. 670-3.

55. Mottaghi-Dastjerdi N, Soltany-Rezaee-Rad M, Sepehrizadeh Z, Roshandel G, Ebrahimifard F, and Setayesh N. Genome Expression Analysis by Suppression Subtractive Hybridization Identified Overexpression of Humanin, a Target Gene in Gastric Cancer Chemoresistance. Daru : Journal of Faculty of Pharmacy, Tehran University of Medical Sciences. 2014; 22(1): p. 14.

56. Omar NN, Tash RF, Shoukry Y, and ElSaeed KO. Breaking the Ritual Metabolic Cycle in Order to
Save Acetyl Coa: A Potential Role for Mitochondrial Humanin in T2 Bladder Cancer Aggressiveness. Journal of the Egyptian National Cancer Institute. 2017; 29(2): p. 69-76.

57. Surampudi P, Chang I, Lue Y, Doumit T, Jia Y, Atienza V, et al. Humanin Protects against Chemotherapy-Induced Stage-Specific Male Germ Cell Apoptosis in Rats. Andrology. 2015; 3(3): p. 582589.

58. Jia Y, Ohanyan A, Lue YH, Swerdloff RS, Liu PY, Cohen P, et al. The Effects of Humanin and Its Analogues on Male Germ Cell Apoptosis Induced by Chemotherapeutic Drugs. Apoptosis : An International Journal on Programmed Cell Death. 2015; 20(4): p. 551-61.

59. Eriksson E, Wickstrom M, Perup LS, Johnsen JI, Eksborg S, Kogner P, et al. Protective Role of $\mathrm{Hu}$ manin on Bortezomib-Induced Bone Growth Impairment in Anticancer Treatment. Journal of the National Cancer Institute. 2014; 106(3): p. djt459.

60. Cohen P. New Role for the Mitochondrial Peptide Humanin: Protective Agent against Chemotherapy-Induced Side Effects. Journal of the National Cancer Institute. 2014; 106(3): p. dju006.

61. Zuccato C, Moreno Ayala M, Gottardo M, Pidre M, Asad A, Nicola Candia A, et al. Role of Mitochondrial Peptide Humanin in the Response of Experimental Breast Cancer to Chemotherapy. Proceedings of the American Association for Cancer Research Annual Meeting. Cancer Research. 2018; 78(12 (Suppl)): p. 1951.

62. Gray GK, McFarland BC, Nozell SE, and Benveniste EN. Nf-Kappab and Stat3 in Glioblastoma: Therapeutic Targets Coming of Age. Expert Review of Neurotherapeutics. 2014; 14(11): p. 1293-306.

63. Elizalde PV, Cordo Russo RI, Chervo MF, and Schillaci R. Erbb-2 Nuclear Function in Breast Cancer Growth, Metastasis and Resistance to Therapy. Endocrine-related cancer. 2016; 23(12): p. T243-T257. 
64. Banerjee $\mathrm{K}$ and Resat $\mathrm{H}$. Constitutive Activation of Stat3 in Breast Cancer Cells: A Review. International Journal of Cancer. 2016; 138(11): p. 2570-8.

65. Li S, Tian J, Zhang H, Zhou S, Wang X, Zhang L, et al. Down-Regulating Il-6/Gp130 Targets Improved the Anti-Tumor Effects of 5-Fluorouracil in Colon Cancer. Apoptosis : An International Journal on Programmed Cell Death. 2018; 23(5-6): p. 356374.

66. Xiao J, Howard L, Wan J, Wiggins E, Vidal A, Cohen $\mathrm{P}$, et al. Low Circulating Levels of the Mitochondrial-Peptide Hormone Shlp2: Novel Biomarker for Prostate Cancer Risk. Oncotarget. 2017; 8(55): p. 94900-94909.

67. Kim SJ, Mehta HH, Wan J, Kuehnemann C, Chen J, Hu JF, et al. Mitochondrial Peptides Modulate Mitochondrial Function During Cellular Senescence. Aging. 2018; 10(6): p. 1239-1256.

68. Schosserer M, Grillari J, and Breitenbach M. The Dual Role of Cellular Senescence in Developing Tumors and Their Response to Cancer Therapy. Frontiers in Oncology. 2017; 7: p. 278.

69. Lasry A and Ben-Neriah Y. Senescence-Associated Inflammatory Responses: Aging and Cancer Perspectives. Trends in Immunology. 2015; 36(4): p. 217-28.

70. Campisi J, Andersen JK, Kapahi P, and Melov S. Cellular Senescence: A Link between Cancer and Age-Related Degenerative Disease? Seminars in Cancer Biology. 2011; 21(6): p. 354-9.

71. Freund A, Orjalo AV, Desprez PY, and Campisi J. Inflammatory Networks During Cellular Senescence: Causes and Consequences. Trends in Molecular Medicine. 2010; 16(5): p. 238-46.

72. Chin YP, Keni J, Wan J, Mehta H, Anene F, Jia Y, et al. Pharmacokinetics and Tissue Distribution of Humanin and Its Analogues in Male Rodents. Endocrinology. 2013; 154(10): p. 3739-44.

73. Sari Y, Chiba T, Yamada M, Rebec GV, and Aiso S. A Novel Peptide, Colivelin, Prevents Alcohol-Indu- ced Apoptosis in Fetal Brain of C57bl/6 Mice: Signaling Pathway Investigations. Neuroscience. 2009; 164(4): p. 1653-64.

74. Lalatsa A, Schatzlein AG, and Uchegbu IF. Strategies to Deliver Peptide Drugs to the Brain. Mol Pharm. 2014; 11(4): p. 1081-93.

75. Yamada M, Chiba T, Sasabe J, Terashita K, Aiso S, and Matsuoka M. Nasal Colivelin Treatment Ameliorates Memory Impairment Related to Alzheimer's Disease. Neuropsychopharmacology. 2008; 33(8): p. 2020-32.

76. Wu H, Li J, Zhang Q, Yan X, Guo L, Gao X, et al. A Novel Small Odorranalectin-Bearing Cubosomes: Preparation, Brain Delivery and Pharmacodynamic Study on Amyloid-Beta(2)(5)(-)(3)(5)-Treated Rats Following Intranasal Administration. Eur J Pharm Biopharm. 2012; 80(2): p. 368-78.

77. Logan IS. Pseudogenization of the Humanin Gene Is Common in the Mitochondrial DNA of Many Vertebrates. Zoological Research. 2017; 38(4): p. 198-202. 